\title{
Effect of An Educational Program on Lifestyle Modification for Patients Undergoing Laparoscopic Sleeve Gastrectomy Surgery
}

\author{
${ }^{1}$ Hala A. El-Maghawry, ${ }^{2}$ Hanaa S. Said, ${ }^{3}$ Mohammed F. Amin, ${ }^{3}$ Ahmed M. Yehia, \\ ${ }^{1}$ Hanaa A. Nofal \\ ${ }^{1}$ Department of Community, Environmental and Occupational Medicine, ${ }^{2}$ Department of \\ Family Medicine, ${ }^{3}$ Department of General Surgery, Faculty of Medicine, Zagazig \\ University, Egypt.
}

\begin{abstract}
Background: Laparoscopic sleeve gastrectomy surgery was viewed as an effective therapy for morbid obesity. The lifestyle of obese patients must be changed to complete surgery success and sustain a permanent loss of weight without recurrence.

Objectives: To evaluate the effect of an instructional guide on lifestyle modification for morbidly obese patients undergoing sleeve gastrectomy surgery at Zagazig University Hospitals. Method: An interventional study was conducted at outpatients' clinic for obesity at Zagazig University Hospitals in stages that included assessment of patients' knowledge regarding lifestyle modification after laparoscopic sleeve gastrectomy surgery. All patients were clinically examined, investigations were done. Then applying a health education program about lifestyle modification after laparoscopic sleeve gastrectomy surgery. Reassessment of patients' knowledge about lifestyle modification following laparoscopic sleeve gastrectomy surgery, re-examining all patients post-operatively to evaluate the intervention. The post-operative assessments: 1-month post-surgery, 6 months post-surgery and 12 months post-surgery were done. Results: A total sample of 38 patients with a mean age $34.6 \pm 5.7$ years. Thirty-nine (39) co-morbidities were identified among them, $56.4 \%$ of them were resolved. The majority of patients (94.8\%) had unsatisfactory knowledge regarding lifestyle modification after laparoscopic sleeve gastrectomy surgery before applying health education program, while after applying health education program the patients' knowledge was satisfactory (81.6\%). Laparoscopic sleeve gastrectomy accompanied by lifestyle modification achieved a marked reduction in body weight and body mass index. Conclusion: Lifestyle modification has an additive role in weight loss with surgical intervention.
\end{abstract}

Key words: Sleeve gastrectomy - lifestyle modification - Health education- Physical activityObesity.

Corresponding author: Hala Ahmed El-Maghawry Email halamf@yahoo.com

\section{Introduction}

Excess weight and obesity epidemic poses a great challenge to the prevention of diseases and health across the life course throughout the world. The prevalence of obesity and overweight is still growing among adults worldwide. ${ }^{1}$ It is a multifactorial complex disease, with genetic, behavioral, environmental and socioeconomic roots. Obese patients are more susceptible to coronary heart disease, type 2 diabetes and mental health problems like depression, anxiety, eating disorders and mortality. ${ }^{2}$

Bariatric surgery has been shown to be significantly superior to intensive medical approaches as regarding treatment options for obesity. It was proven to be a successful procedure for morbidly obese patients reducing about $30 \%$ of their total weight, as well as improving obesity-related comorbidities. ${ }^{3} \quad$ Laparoscopic sleeve gastrectomy (LSG) is gaining worldwide 
popularity. It means surgical excision of the gastric body and fundus, leaving a narrow, tubular stomach along the lesser curvature of the stomach. ${ }^{4}$ Patients' lifestyle must be modified to complete success of surgery and keep permanent weight loss without recurrence. Possible important predictor of weight loss after surgery is adherence to post-surgery behavioral guidelines during the first post-operative years. ${ }^{5}$

Increased physical activity following bariatric procedures is an effective longterm weight loss management strategy. Physical activity has a range of long, short-term health effects like improving cardiovascular diseases, obesity, diabetes and hypertension, some cancer types, depression, improvement of bone, energy balance, as well as regulation of body weight. Increasing physical activity postoperatively is also essential for bariatric patients for enhancing weight loss, preserving muscle mass and decreasing the possibility of weight regain. ${ }^{6}$ Bariatric patients who do not comply with the recommended guidelines of lifestyle modification post-operatively are at risk for regaining their previous weight and development of protein, iron, vitamin B12, folate, calcium deficiencies. Therefore, it is important for patients to be fully aware about the required dietary changes and about the level of post-operative physical activity. ${ }^{7}$ Previous studies on laparoscopic sleeve gastrectomy (LSG) concluded that providing a lifestyle modification educational program (nutritional education and increasing physical activity) after LSG can significantly improve patients' knowledge and practice as better pre-operative knowledge would result in more weight loss postoperatively. ${ }^{8}$

To our knowledge, no research has nvestigated the relationship between preoperative knowledge and weight loss after laparoscopic sleeve gastrectomy surgery in Zagazig University Hospitals.
Taken together the lack of postoperative data and recommendations from systematic reviews ${ }^{9}$ provide a strong rational for conducting a study to evaluate Effect of an educational program on lifestyle modification for patients undergoing laparoscopic sleeve gastrectomy surgery.

\section{Objectives}

To evaluate the effect of instructional guide on lifestyle modification (nutritional education and physical activity) for patients with morbid obesity undergoing sleeve gastrectomy surgery in Zagazig University Hospitals and that was achieved through: (1) Comparing the level of patients' knowledge as regarding lifestyle modification (nutritional education and physical activity) after LSG pre and postoperative. (2) Evaluating the effect of an instructional guide on patients' lifestyle modification post sleeve gastrectomy surgery as regarding body weight, comorbidities remission and improvement on lipid profile by evaluating the post-operative changes such as: Excess weight loss percentage (\%EWL) and excess BMI loss percentage (\%EBMIL) at 1, 6 and 12 months post- operative relative to baseline pre- operative weight; Comorbidities associated with obesity (e.g. type 2 diabetes, hypertension, obstructive sleep apnea and osteoarthritis at 1,6 and 12 months post-operative comparable to pre-operative.

\section{Method}

An intervention study design was performed at outpatients' clinic for obesity at Zagazig University Hospitals.

A total coverage of all morbidly obese patients undergoing laparoscopic sleeve gastrectomy surgery (LSG) at Zagazig University Hospitals from the first of October 2017 to the end of September 2019 (from recruiting first patient to end of follow-up of last patient). A total of 63 cases were recruited in the beginning of the present study. After that, 25 cases 
were excluded from the recruit, (9 of them developed post-operative complications such as leakage and hemorrhage, in addition of 16 cases who failed to do follow- up visits). So, the net number of cases was 38 (9 males and 29 females, whose age ranged from 25 to 53 years). The cases were regularly monitored post-operatively over 12 months for weight reduction and metabolic changes through measurement of BMI, blood sugar levels, and total body cholesterol levels.

Inclusion criteria: All patients were fit for surgery and met criteria qualifying for laparoscopic sleeve gastrectomy surgery: patients had BMI (body mass index) greater than $35 \mathrm{~kg} / \mathrm{m} 2$ with obesity related diseases (osteoarthritis, type 2 diabetes, obstructive sleep apnea and hypertension) or patients had BMI greater than $40 \mathrm{~kg} / \mathrm{m} 2$ and have already failed previous diet trials, exercise and medications.

Exclusion criteria: Complicated cases (Leakage, hemorrhage and pulmonary embolism) was excluded due to long hospitalization

Tools of the study and procedures: Preoperative assessment: Pretest for assessment of patients' knowledge, regarding lifestyle modification after laparoscopic sleeve gastrectomy surgery, then applying a health education program about lifestyle modification after laparoscopic sleeve gastrectomy surgery (nutritional modification and physical activity) in the outpatient clinic of obesity one week before surgery.

Tool (I): $\quad$ semi-instructured questionnaire: to evaluate effect of educational program on patients' knowledge about lifestyle modification (nutritional education and physical activity) following LSG after reviewing the related literature. It has been written in simple Arabic language. It contains two sections.

Section I: socio-demographic data of patients and medical history to identify presence of chronic diseases that related to morbid obesity (comorbidities), other weight reduction programs before laparoscopic sleeve gastrectomy.

Section II: Pre/post patients' knowledge assessment questionnaire containing closed ended questions about the following items: Morbid obesity definition, causes and complications (4 questions with only one correct answer, total score= 4). Laparoscopic sleeve gastrectomy definition, benefits, preoperative preparation and postoperative problems i.e. redundant skin, sense of hunger, return to work and possibility of pregnancy (4 questions, some contains more than one correct answer so, total score $=6$ ). Life style modification postoperatively: (a) Nutritional adjustment of dietary principles (energy needed in Kcals, protein and fat contents, meal frequency, pre-operative liquid diet, post-operative dietary phases, types of food after the surgery and nutrition symptoms such as dehydration, nausea, and vomiting) (6 questions, some contains more than one correct answer so, total score $=23) .{ }^{10}(\mathrm{~b})$ Supplementation of vitamins and minerals (1 question with 3 correct answers so, total score $=3) .{ }^{10}$ (c) Physical activity and continuous monitoring of weight ( 2 questions, with more than one correct answer so, total score $=5)$. (d) Follow up visits and their importance (2 questions with only one correct answer, total score $=2$ ).

Questions with more than one correct answer: Each chosen answer took 1 degree. The total score of all items of knowledge was 43 degree. Adequacy of knowledge was as follows: cut off point is $60 \%$ whereas: $\geq 60 \%$ of the total score was considered as satisfactory knowledge and $<60 \%$ of the total score was considered as unsatisfactory knowledge. ${ }^{11}$ The questionnaire was validated by panels of experts in community and family medicine departments. The questionnaire was 
translated from English to Arabic by expert translator, then back-translated by another expert translator, and finally, the original and translated versions were compared by another bilingual expert to ensure validity. The reliability coefficient test (Cronbach's alpha) was $=0.72$ for all questions. A pilot study (10\% of the total sample) was implemented to estimate the time needed to obtain the required informations, and there was no administrative or technical obstacles founded (the total sample included the pilot sample).

Tool (II): anthropometric measurements: A specialized bariatric surgery team of general surgeons, anesthesiologists, endocrinologists, pulmonologists, cardiologists and psychiatrists carefully examined all patients before the operation. Pre-operative screening included medical examination, anthropometric measurements (Weight and height), nutritional evaluation, abdominal ultrasonography and upper GIT endoscopy. Electronic balance was used to measure weight $(\mathrm{Kg})$ in the standing position with participants wearing light clothes and without shoes. $100 .^{12}$

Table (1): Age, Sex and Body Mass Index of The Study Participants at The Outpatient Clinic of Obesity at Zagazig University Hospital, 2019

\begin{tabular}{|c|c|}
\hline $\begin{array}{l}\text { Variables } \\
\text { No. }(\%)\end{array}$ & No. $=38$ \\
\hline $\begin{array}{l}\text { Age at surgery (year) No. } \\
(\%):\end{array}$ & \\
\hline $25-$ & $13(34.3)$ \\
\hline 35 - & $22(57.8)$ \\
\hline $40+$ & $3(7.9)$ \\
\hline Mean \pm SD $^{*}$ & $34.6 \pm 5.7$ \\
\hline Sex: & \\
\hline - Male & $9(23.6)$ \\
\hline - Female & $29(76.4)$ \\
\hline $\begin{array}{l}\text { Preoperative } \\
(\mathrm{kg} / \mathrm{m} 2): \\
\text { Mean } \pm \text { SD }\end{array}$ & $39.2 \pm 2.6$ \\
\hline $\begin{array}{l}\text { *SD: Standard Deviation, BMI } \\
\text { Index. } \\
\text { Likewise, height }(\mathrm{m}) \text { was } \\
\text { using length measurin }\end{array}$ & $\begin{array}{l}\text { : Body Mass } \\
\text { measured by } \\
\text { ag device }\end{array}$ \\
\hline
\end{tabular}

(stadiometer). Body mass index (BMI) was calculated: weight in kilograms divided by height in meters squared $(\mathrm{kg} / \mathrm{m} 2)$. To evaluate evolution of patients in losing weight, Percentage of changes of weight and BMI were calculated using the following formulas: Excess weight Loss percentage (\%EWL): $100 \% \times$ (preoperative $\mathrm{W}$ - current $\mathrm{W}$ )/ (preoperative W - ideal W). Excess BMI loss percentage (\%EBMIL): $100 \% \times$ (preoperative BMI - current BMI)/ (preoperative BMI - ideal BMI). Ideal weight weight corresponding to $85^{\text {th }}$ percentile for the patient's age, gender, and height. Percentage of change: ((after value - before value) / before value) *

Tool (III): Biochemical follow up: Complete blood count (CBC), fasting Blood Sugar (FBS), blood pressure (mmHg), and Lipid profile: Low Density Lipoprotein (LDL), High Density Lipoprotein (HDL), total cholesterol (TC) and Triglycerides (TGs).

Table (2): Comorbidities in the study participants at the outpatient clinic of obesity at Zagazig University Hospital, 2019

\begin{tabular}{lll}
\hline \multicolumn{2}{c}{ Variables } & No. (\%) \\
\hline $\begin{array}{l}\text { Presence of co-morbidities } \\
\text { (No.=38): }\end{array}$ & & 34 \\
- Yes & & $(89.5)$ \\
- No & & $4(10.5)$ \\
\hline
\end{tabular}

Type of co-morbidities:

- Diabetes (No.= 10)

- Not changed $2(20.0)$

- controlled $8(80.0)$

$\begin{array}{ll}\text { Hypertension }(\text { No. }=9) & 9) \\ -\quad \text { Not changed } & 1(11.1)\end{array}$

- controlled $8(88.9)$

- Obstructive sleep apnea (No.= 11) $2(18.1)$

- Not changed $9(81.9)$

- controlled

- Osteoarthritis (No.= 9) $3(33.4)$

- Not changed $6(66.6)$

- controlled

The intervention phase: Health education intervention program about lifestyle modification (nutritional education and physical activity) following sleeve gastrectomy surgery by 
laparoscope was performed in Zagazig University Hospitals. Topics were covered in each educational session to answer pretest questions in addition to items about: Preoperative preparation of the operation, postoperative problems like nausea and vomiting, dehydration, constipation, intolerance to food, and hypoglycemia were discussed. Strategies for prevention were addressed. General nutrition topics: pre- and post-operative digestion, post-operative dietary development, eating and drinking strategies, and protein supplementations. Operational definition of the required physical activity: The definition, level and time of physical activity have been illustrated to identify physical activity in different domains "Work, Transport and Recreation time". Physical activity was defined as any bodily movement produced by skeletal muscles that require energy expenditure. The term "physical activity" was clarified not to be mistaken with "exercise". Physical activity includes exercise as well as other activities which involve bodily movement and are done as part of playing, working, active transportation, house chores and recreational activities. At least a minimum of 60 minutes of moderate intensity physical activity is required daily (in one block or it can also be separated) for long-term maintenance of significant weight loss. ${ }^{13}$

The educational intervention program was given at obesity outpatient clinic of Zagazig University Hospitals individually prior to the operation (in the pre-intervention phase one week before surgery). The educational material was organized in a standardized way and disseminated as an information brochure that briefly clarified and explained each topic discussed in the session. On average, the duration of each session was nearly 40 minutes. To be easily understood, the program was written in Arabic language. The bariatric surgery team performed operations on the scheduled date.

One month post-operative assessment: To evaluate the intervention, this phase was performed one month after the surgery. Reassessment of patients' knowledge about lifestyle modification following laparoscopic sleeve gastrectomy surgery through the same questionnaire which was used in the preoperative phase. The bariatric surgery team carefully re-examine all patients. Nutritional evaluation, anthropometric measurements, upper gastrointestinal endoscopy and abdominal ultrasound were included in post-operative assessments. Blood Pressure, complete blood count, blood sugar, lipid profile, and chest $\mathrm{x}$-ray were screened postoperatively.

6 months post-operative assessment: was performed with all steps done in the first post-operative assessment.

12 months post-operative assessment was performed with all steps done in the first post-operative assessment.

\section{Ethical approval}

Approval by an Institutional Review Board (IRB) for medical research ethics, Zagazig University, Faculty of Medicine was obtained before implementation of the study (ZU-IRB 5732). Prior to implementation of the study, an official approval for the implementation of the study was obtained from the director of Zagazig University outpatient clinics to the outpatient clinic of obesity at Zagazig University Hospital after explaining the aim of the research to get the permission for data collection. An informed consent was obtained from each participant prior to data collection which addresses the confidentiality of data collected and assuring that participation in the study is completely voluntary.

\section{Statistical Analysis}

Data analysis was performed using the software SPSS (Statistical Package for the Social Sciences) version 20. Categorical variables were described 
using their absolute frequencies. McNemar's test was used to analyze paired qualitative data. Repeated measures ANOVA and Bonferroni posthoc test were used to analyze paired quantitative data on repeated measures. The level of statistical significance was set at 5\% $(P<0.05)$.

\section{Results}

The study sample comprised of 38 patients, their mean age $34.6 \pm 5.7$ years, as shown in Table (1) which revealed that more than half of them $(57.8 \%)$ in the age group from 35 to 40 years old. About three quarters of them $(76.4 \%)$ were women.

Out of the 38 patients, $89.5 \%$ of patients reported co-morbidities (Table 2).

Vast majority of patients $(94.8 \%)$ had unsatisfactory knowledge about life style modification after laparoscopic sleeve gastrectomy surgery before applying health education program, while the knowledge of patients was satisfactory after applying the health education program in the $1^{\text {st }}, 2^{\text {nd }}$ and $3^{\text {rd }}$ postoperative assessments (97.4\%, 89.4\% and $81.6 \%$ respectively). And this difference was statistically significant $(\mathrm{P}<0.05)$ (Table 3).

Regarding weight loss of cases, a significant reduction in BMI and body weight was achieved by lifestyle modification following LSG. The mean weight was $19.8 \pm 10.3 \mathrm{~kg}$, the mean BMI was $25.7 \pm 1.7$ after 1 year of follow up (Table 4). Table 5 showed significant difference within the study groups as regarding weight loss descriptive after LSG (weight, BMI, \% EWL, \% EBMIL).

The changes in lipid profile levels (plasma glucose, LDL, TC, and TGs levels) were statistical significant in the post-operative measurements $(\mathrm{P}<0.05)$ (Table 6). Table

Table (3): Total Patients' Knowledge Regarding Life Style Modification after Laparoscopic Sleeve Gastrectomy Surgery at The Outpatient Clinic of Obesity at Zagazig University Hospital, $2019(\mathbf{n}=38)$

\begin{tabular}{lllllll}
\hline \multirow{2}{*}{ Study phases } & \multicolumn{2}{c}{ Total patients' knowledge } & \multicolumn{3}{c}{$\begin{array}{c}\text { McNemar's test } \\
\text { P value }\end{array}$} \\
\cline { 2 - 6 } & $\begin{array}{c}\text { Satisfactory } \\
\text { No. (\%) }\end{array}$ & $\begin{array}{c}\text { Unsatisfactory } \\
\text { No. (\%) }\end{array}$ & P1 $\dagger$ & P2 $\ddagger$ & P3§ \\
\hline Pre-operative assessment & $2(5.2)$ & $36(94.8)$ & & & \\
1 month post-operative assessment & $37(97.4)$ & $1(2.6)$ & $0.001^{*}$ & $0.001^{*}$ & $0.001^{*}$ \\
6 months post-operative assessment & $34(89.4)$ & $4(10.6)$ & & & \\
\hline 12 months post-operative assessment & $31(81.6)$ & $7(18.4)$ & & & \\
\hline
\end{tabular}

* Statistical significance: $\mathrm{p}<0.05$ is considered statistically significant. $\dagger$ P1: Difference between Preintervention assessment and $1^{\text {-month }}$ post-operative assessment: $\$$ P2: Difference between Pre-intervention assessment and 6 months post-operative assessment. § P3: Difference between Pre-intervention assessment and 12 months post-operative assessment

Table (4): Weight Loss Descriptive after LSG at The Outpatient Clinic of Obesity at Zagazig University Hospital, 2019 (Mean \pm SD):

\begin{tabular}{|c|c|c|c|c|c|}
\hline \multirow{3}{*}{ Variables } & \multicolumn{4}{|c|}{ Study phases } & \multirow{3}{*}{$\begin{array}{l}\text { F test" } \\
\text { P value }\end{array}$} \\
\hline & \multirow{2}{*}{$\begin{array}{l}\text { Pre-operative } \\
\text { assessment }\end{array}$} & \multicolumn{3}{|c|}{ post-operative assessment } & \\
\hline & & 1 month & 6 months & 12 months & \\
\hline Weight (Kg) & $46.8 \pm 19.3$ & $40.7 \pm 19.1$ & $31.3 \pm 13.5$ & $19.8 \pm 10.3$ & $0.001 * *$ \\
\hline $\mathbf{B M I}^{\dagger}$ & $39.2 \pm 2.6$ & $32.3 \pm 3.4$ & $28.8 \pm 2.3$ & $25.7 \pm 1.7$ & $<0.001 * *$ \\
\hline$\% \mathbf{E W L}^{*}$ & 0 & $27.9 \pm 1.6$ & $47.2 \pm 2.3$ & $60.1 \pm 2.8$ & $<0.001 * *$ \\
\hline$\%$ EBMIL $^{\S}$ & 0 & $24.1 \pm 1.6$ & $56.7 \pm 1.4$ & $72.8 \pm 9.5$ & $<0.001 * *$ \\
\hline $\begin{array}{l}\text { Percent change in } \\
\text { mean BMI }\end{array}$ & & -17.6 & -26.5 & -34.4 & \\
\hline
\end{tabular}


$*$ Repeated measures ANOVA. $†$ BMI: body mass index. $\$ \%$ EWL: percentage of excess weight loss. $\S$ EBMIL percentage of excess BMI loss. ** Statistical significant differences $(\mathrm{P}<0.05)$.

Table (5): Bonferroni Post-Hoc Test to Detect Significant Difference within The Study Groups at The Outpatient Clinic of Obesity at Zagazig University Hospital, 2019

\begin{tabular}{lrrrrrr}
\multicolumn{1}{c}{ Variables } & P1 & P2 & P3 & \multicolumn{1}{c}{ P4 } & P5 & \multicolumn{1}{c}{ P6* } \\
\hline Weight (Kg) & 0.3 & $<0.001$ & $<0.001$ & 0.04 & $<0.001$ & 0.01 \\
\hline BMI & $<0.001$ & $<0.001$ & $<0.001$ & $<0.001$ & $<0.001$ & $<0.001$ \\
\hline \% EWL & $/$ & $/$ & $/$ & $<0.001$ & $<0.001$ & $<0.001$ \\
\hline \% EBMIL & $/$ & $/$ & $/$ & $<0.001$ & $<0.001$ & $<0.001$ \\
\hline
\end{tabular}

P1: Pre-operative assessment vs. 1-month post-operative assessment. P2: Pre-operative assessment vs. 6 months post-operative assessment. P3: Pre-operative assessment vs. 12 months post-operative assessment. P4: 1-month post-operative assessment vs. 6 months post-operative assessment. P5: 1-month post-operative assessment vs. 12 months post-operative assessment. P6: 6 months post-operative assessment vs. 12 months post-operative assessment.

Table (6): Metabolic effects after LSG at the outpatient clinic of obesity at Zagazig University Hospital, 2019 (Mean \pm SD)

\begin{tabular}{|c|c|c|c|c|c|}
\hline \multirow{3}{*}{ Variables } & \multicolumn{4}{|c|}{ Study phases } & \multirow{3}{*}{$\begin{array}{l}\text { F test } \\
\text { P value }\end{array}$} \\
\hline & \multirow{2}{*}{$\begin{array}{c}\text { Pre-operative } \\
\text { assessment }\end{array}$} & \multicolumn{3}{|c|}{ Post-operative assessment } & \\
\hline & & 1 month & 6 months & 12 months & \\
\hline Plasma glucose (mmoL) & $7.1 \pm 1.3$ & $6.4 \pm 1.9$ & $6.1 \pm 1.7$ & $3.1 \pm 0.2$ & $<0.001^{*}$ \\
\hline HDL (mg/dL) ${ }^{\dagger}$ & $44.2 \pm 12.7$ & $45.3 \pm 13.1$ & $48.2 \pm 9.2$ & $49.2 \pm 10.4$ & 0.18 \\
\hline 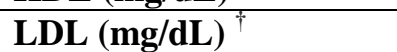 & $113.6 \pm 30.2$ & $113.1 \pm 28.5$ & $109.8 \pm 25.6$ & $90.8 \pm 24.1$ & $<0.001^{\ddagger}$ \\
\hline TC $(\mathrm{mg} / \mathrm{dL})^{\dagger}$ & $186.5 \pm 35.1$ & $181.7 \pm 31.4$ & $176.6 \pm 30.3$ & $153.3 \pm 26.9$ & $<0.001^{+}$ \\
\hline TGs (mg/dL) & $154.3 \pm 58.7$ & $110.7 \pm 43.1$ & $103.8 \pm 28.1$ & $102.3 \pm 31.4$ & $<0.001^{\ddagger}$ \\
\hline
\end{tabular}

* Repeated measures ANOVA. $\dagger$ HDL (High-density lipoprotein), LDL (Low-density lipoprotein), TC (Total cholesterol), TGs (Triglycerides). $\$$ Statistically significant differences $(\mathrm{P}<0.05)$.

Table (7): Bonferroni Post-Hoc Test to Detect Significant Difference within The Study Groups at The Outpatient Clinic of Obesity at Zagazig University Hospital, 2019

\begin{tabular}{lrrrrrr}
\multicolumn{1}{c}{ Variables } & \multicolumn{1}{c}{ P1 } & \multicolumn{1}{c}{ P2 } & \multicolumn{1}{c}{ P3 } & P4 & \multicolumn{1}{c}{ P5 } & \multicolumn{1}{c}{ P6* $^{*}$} \\
\hline Plasma glucose (mmoL) & 0.14 & 0.01 & $<0.001$ & 0.7 & $<0.001$ & $<0.001$ \\
\hline LDL (mg/dL) & 1 & 0.9 & 0.02 & 0.9 & 0.02 & 0.01 \\
\hline TC (mg/dL) & 0.9 & 0.5 & $<0.001$ & 0.8 & 0.01 & 0.01 \\
\hline TGs (mg/dL) & 0.001 & $<0.001$ & $<0.001$ & 0.8 & 0.8 & 0.9 \\
\hline
\end{tabular}

*P1: Pre-operative assessment vs. 1-month post-operative assessment. P2: Pre-operative assessment vs. 6 months post-operative assessment. P3: Pre-operative assessment vs. 12 months post-operative assessment. P4: 1-month post-operative assessment vs. 6 months post-operative assessment. P5: 1-month post-operative assessment vs. 12 months post-operative assessment. P6: 6 months post-operative assessment vs. 12 months post-operative assessment

7 showed the difference within the study groups as regarding metabolic effects after LSG. There were statistically significant differences in the 12-months post-operative measurements as regarding plasma glucose, LDL, TC (Table 7).

\section{Discussion}

Bariatric procedures are the ideal current choice for patients with morbid obesity because its results in sustained weight loss and mortality reduction. LSG avoids intestinal bypass so, it became the leading bariatric surgery technique due to its simplicity and efficiency. ${ }^{14}$

Numerous case-control and retrospective studies have shown that LSG is equal to or more effective than other bariatric procedures in terms of weight loss and improvements in short-term obesityrelated chronic conditions. ${ }^{15}$ 
Postoperative weight reduction and knowledge about lifestyle modification were examined in the 38 patients submitted for LSG surgery in current study at 1, 6 and 12 months following the surgery. More than half of them were in the $3^{\text {rd }}$ decade $(57.8 \%)$ with mean age was $34.6 \pm 5.7$ years, the percentage of women was almost three times higher than that of men (76.4: 23.6). Preoperative mean BMI was $39.2 \pm 2.6$ $\mathrm{kg} / \mathrm{m}^{2}$ (table 1).

With increasing prevalence of obesity, weight management becomes a vital but a challenging feature. The causes are the increased rates of comorbidities and more specifically, the resulting physical and cognitive disability. In the current study, $89.5 \%$ reported co-morbidities. Of these medical conditions, most of them have been controlled $(80.8 \%$ of diabetes cases, $88.9 \%$ of hypertension cases, $81.9 \%$ of obstructive sleep apnea cases and $66.6 \%$ of osteoarthritis cases) (Table $2)$. This result is nearly agreeing with kirkil et al who found that 772 comorbidities were reported in 1138 adult patients underwent LSG. Of these medical conditions, $84.4 \%$ have been controlled. $^{16}$

Regarding knowledge about life style modification (nutritional education and exercise) following laparoscopic sleeve gastrectomy surgery, McNemar's test was used for assessing the knowledge; it showed statistically significant difference between pre and post-operative assessments $\quad(\mathrm{p}<$ 0.005). It was interesting that the knowledge of patients had increased over time, even if it was measured 30 days after surgery (Table 3 ). This can be explained by the fact that pre-and post-operative training sessions offer practical knowledge, expertise and support to the bariatric patient in order to make the necessary changes and reduce weight. This is in line with a Canadian study that reported that the key hypothesis about post-operative knowledge improvements was performing educational and training sessions pre-operatively. ${ }^{10}$ A research by McGrice and Don Paul also found that after bariatric surgery, patients who had at least 12 follow up visits through the first six months following the surgery had significantly improved weight loss re sults. ${ }^{17}$

Results of the present study advocate the efficacy of LSG in weight loss. Patients are tested for preoperative weight, 1, 3, and 12 months postoperative weight reduction. Regarding BMI, from starting values $39.2 \pm 2.6 \mathrm{~kg} / \mathrm{m} 2$ mean BMI prior to surgery and educational program, an important decrease was observed to a mean value of $25.7 \pm 1.7 \mathrm{~kg} / \mathrm{m} 2$ was observed 1 year after surgery (table 4). This is comparable to findings reported in Norway by Gjessing et al. who reported a significant reduction in preoperative BMI for all patients three months after operation and a more reduction after 1 year. ${ }^{18}$

It is considered that reaching $50 \%$ of EWL is proved to be a predictor of improvement of health and associated quality of life. ${ }^{18}$ After 12 months from surgery in the current study, mean $\%$ EWL values was more than the $60 \%$ $(60.1 \pm 2.8 \%)$. Along with \%EWL values, \%EBMIL values also increased from a mean $\%$ value of $24.1 \pm 1.6$ to $72.8 \pm 9.5$ after 12 months following surgery (Table 4). Correspondingly, Golomb et al. reported a statistical significant reduction in EWL percentage within one and three years of follow-up $\left(\mathrm{P}=\right.$ 0.007). ${ }^{19}$ Monzo-Beltrana et al. reported similar findings following LSG, where 114 patients followed up for 5 years. Mean EWL was more than $65 \%$ in the first 3 years and declined to $45.3 \%$ through 5 years. This is documented through self-reporting of patients at the follow-up visits, where they asked whether they had improved their lifestyle significantly as regarding the pattern of eating habits and physical activity. Implementation of good dietary habits 
and increased physical exercise or, in other words, significant lifestyle modification is the base for LSG's success. ${ }^{20}$ However, the results of the current study not agree with results of Al-Sabah et al. study, who documented that the highest percentage of EWL was reached at 1 month after LSG (73.8 \pm 26.4) ${ }^{21}$ As expected, along the study, all patients showed a progressive decrease of weight as well as of its derived variables: BMI, \%EWL and \%EBMIL. There was statistical significant differences when comparing the four phases of the study $(\mathrm{P}<0.05)$. Reduction of weight was observed to continue decreasing at 1,6 and 12 months-time after bariatric surgery (table 5). It may be explained by the fact that, specifically in the short and mid-term, LSG is a very effective restrictive technique. It is inevitable that a person undertakes a restrictive procedure will not be able to achieve long-term weight loss without restricting the intake of dietary calories or increasing physical activity and energy consumption.

In conjugation with weight loss, all metabolic parameters analyzed also showed significant improvement. Plasma glucose level and lipid profile (TC, TGs and LDL) had been significantly decreased at 1 year after surgery (Table 6). It may be due to the fact that losing body weight is a clear sign of better health so, normal values were reached within 12 months after surgery. Similar results were stated by Keleidari et al. on FBS and related HbAlc that had been significantly reduced after 1 year of follow-up. ${ }^{22}$

As an indirect result of better nutritional status and mostly due to weight loss, levels of triglycerides levels started to decrease significantly one month after surgery and keeping the decreasing tendency during the study period along with plasma glucose level, LDL, TC levels (table 7). This is in agreement with results of Yildiz et al., where they studied the impact of LSG on the nonWestern population and found that LSG is highly efficient in the postoperative amelioration of hyperlypidemia and diabetes mellitus in the early postoperative follow up periods. ${ }^{23}$

\section{Conclusion}

Lifestyle modification is the base for successful laparoscopic sleeve gastrectomy surgery, as it has an additive role in weight loss with surgical intervention.

\section{Recommendations}

Based on the results of the present study, the authors recommend the following: (1) Such findings highlight the need for a broader strategy to bariatric preoperative treatment, one that encourages the patient to be more responsive to the training methods, behavioral modification, treatment and counseling practiced by registered dietitians and nutrition experts. (2) Cases should be followed-up for longer time than one year. It will be more informative about weight loss, and changes in medical conditions of the participants. (3) Bariatric educational programs must be provided by multidisciplinary team of healthcare providers to ensure consistent and effective weight loss after bariatric procedure by usage of digital tools of communication such as social networking, phone services, and online services to reduce barriers like cost, time, and distance. (4) People who seek weight loss should engage in physical work for at least 60 minutes per day because bariatric patients not involved in physical exercise must integrate repetitive physical work habits into daily routines to sustain weight loss. (5) Overweight and obese people should perform regular psychological, medical, and dietary assessments.

\section{Limitations of the study:}

Larger sample was needed to the study to be extrapolated: we can recruit only an average of five cases per month. This is due to the high cost of the surgical 
procedure, and like any major operation, LSG carries potential health risks in both the short term and long term.

Due to the small sample size, the study design does not include a comparison group. It was preferred to conduct control groups [e.g. surgery alone vs. dual intervention (both surgery \& education)] to clarify the additive role of the educational program on sustained weight loss with surgical intervention not a substitute to it.

The study conducted in a single center (outpatients' clinic for obesity at Zagazig University Hospitals) because it was difficult to conduct the study in a private hospital or clinic.

\section{References}

1. Schiff M, Maria C, Lorraine G, Mary W. Examining Nutrition Knowledge of Bariatric Surgery Patients: What Happens to Dietary Knowledge over Time? OBES SURG 2016; 26:972-982.

2. Adela H, Frank B. The Epidemiology of Obesity: A Big Picture, PMC 2015; 33(7): 673-689.

3. Adams T, Davidson L, Litwin S, Hunt S. Gastrointestinal surgery: cardiovascular risk reduction and improved long-term survival in patients with obesity and diabetes. Curr Atheroscler Rep. 2012; 14(6):606-621.

4. Schiavo L, Scalera G, Pilone V, et al. Patient adherence in following a prescribed diet and micronutrient supplements after laparoscopic sleeve gastrectomy: our experience during 1 year of follow-up. J Hum Nutr Diet. 2017; 30(1):98-104.

5. Jong M, Hinnen C. Bariatric surgery in young adults: a multicenter study into weight loss, dietary adherence, and quality of life. Surgery for Obesity and Related Diseases 2017; 1204-1211.

6. World Health Organization. Global strategy on diet, physical activity and health: physical activity. Geneva, Switzerland: World Health Organization; 2014. Available from:

http://www.who.int/dietphysicalactivity/pa/e n/. Accessed December 22, 2014.

7. Park Y, Kim S. Laparoscopic sleeve gastrectomy as revisional surgery for adjustable gastric band erosion. J.
Laparoendosc. Adv. Surg. Tech. 2014; 24 (9): 593-600.

8. Keren D, Matter I and Lavy A. Lifestyle Modification Parallels to Sleeve Success. Obesity Surgery. 2014; 24: 735-740.

9. Afshar S, Seymour K, Kelly S, et al. Changes in physical activity after bariatric surgery: using objective and self-reported measures. Surgery for Obesity and Related Diseases 2017; 13:474-483.

10.Taube-Schiff M, Chaparro M, Gougeon L, Shakory S. Examining Nutrition Knowledge of Bariatric Surgery Patients: What Happens to Dietary Knowledge over Time? OBES SURG. 2016; 26:972-982.

11.Taukobong N, Myezwa H, Prof Pengpid $\mathrm{S}$, et al. Knowledge, attitude and practice about health promotion amongst physiotherapists in South Africa. SA Journal of Physiotherapy. 2014; 70:2-9.

12. Friedrich C, Carnemolla A, Kingett $H$, Paton B. Protocol for a 1-year prospective, longitudinal cohort study of patients undergoing Roux-en-Y gastric bypass and sleeve gastrectomy: the BARILIFESTYLE observational study. BMJ Open 2018; 8:e020659.

13.World Health Organisation. Global Strategy on Diet, Physical Activity and Health. 2019 [https://www.who.int/dietphysicalactivity/pa/ en/][Accessed 18 November 2019].

14.Erol M, Gures N. Laparoscopic Sleeve Gastrectomy, as an Ultimate Intervention for Fatness Treatment, Make Improved Physiological Changes in Obese Patients. Asian J Biomed Pharmaceut Sci. 2017; 7 (60) 4- 7.

15.American Society for Metabolic \& Bariatric Surgery (ASMBS) Clinical Issues Committee. Updated position statement on sleeve gastrectomy as a bariatric procedure. Surg Obes Relat Dis. 2012;8(3): e21-e26.

16.kirkil C, Aygen E, Korkmaz M, Bozan M. Quality of life after laparoscopic sleeve gastrectomy using baros system. ABCD Arq Bras Cir Dig. 2018; 31(3):e1385.

17.McGrice M, Don Paul K. Interventions to improve long-term weight loss in patients following bariatric surgery: challenges and solutions. Diabetes, Metabolic Syndrome and Obesity: Targets and Therapy 2015; 8: 263-274.

18.Gjessing $\mathrm{H}$, Nielsen $\mathrm{H}$, Mellgren $\mathrm{G}$, Gudbrandsen O. Energy intake, nutritional 
status and weight reduction in patients one year after laparoscopic sleeve gastrectomy 2013; 2:352- 359.

19.Golomb I, David M, Glass A, Keidar A. Long-term Metabolic Effects of Laparoscopic Sleeve Gastrectomy. JAMA Surgery 2015; 150 (11): 1051- 1057.

20.Monzo-Beltrana L, Vazquez-Tarragónb A, Cerdàc C, Garcia-Pereza P. One-year follow-up of clinical, metabolic and oxidative stress profile of morbid obese patients after laparoscopic sleeve gastrectomy. 8-oxo-dG as a clinical marker. Redox Biology 2017; 12: 389-402.
21.Al-Sabah S, Al-Mutawa A, Anderson A, Al-Mutawa M. Evaluation of Nutritional Status Post Laparoscopic Sleeve Gastrectomy-5-Year Outcomes. OBES SURG. 2017; 1- 11.

22.Keleidari B, Mahmoudie M, Anaraki A, et al. Six month-follow up of laparoscopic sleeve gastrectomy. Adv Biomed Res. 2016; 5: 49 .

23. Yildiz B, Katar K, Hamamci O. Efficacy of laparoscopic sleeve gastrectomy for the treatment of obesity in a non- Western society. Eat Weight Disord. 2016; 21: 695699. 